\title{
The Galactic Bulge
}

\author{
B. Barbuy ${ }^{1,2}$ \\ ${ }^{1}$ Universidade de São Paulo, IAG, Rua do Matão 1226, Cidade Universitária, São Paulo 05508-900, Brazil \\ ${ }^{2}$ Email: barbuy@iag.usp.br
}

(Received April 16, 2016; AcCePted May 03, 2016)

\begin{abstract}
The Galactic bulge is the least studied component of our Galaxy. Yet, its formation and evolution are key to understand the formation of the Galaxy itself. Studies on the Galactic bulge have increased significantly in the last years, but still there are many points of controversy. This volume contains several contributions from experts in different aspects of the bulge. Issues discussed include the following: the presence of an old spheroidal bulge, or identification of its old stellar population with the thick disk or halo; fraction of stars younger than $10 \mathrm{Gyr}$ is estimated to be of $<5$ to $22 \%$ depending on method and authors; multiple populations or only a metal-poor and a metal-rich ones; spheroidal or ellipsoidal distribution of RR Lyrae; formation of the bulge from early mergers or from secular evolution of the bar; different methods of mapping extinction; selection and identification of bulge globular clusters.
\end{abstract}

Keywords: Galaxy: bulge

\section{INTRODUCTION}

The formation and evolution of the Galactic bulge has been subject to intense debate in the last $25 \mathrm{yrs}$, as can be seen for example in the proceedings of the IAU Symposium 153, entitled Galactic Bulges, edited by Dejonghe \& Habing (1993). The controversies stem from two types of evidences: (a) Stellar populations: metallicities, abundances, ages, kinematics; (b) morphology of the bulge, in particular, the presence of a bar, and consequently its boxy/peanut shape, and corresponding theoretical simulations.

The evidence from stellar populations indicate that:

\subsection{Age}

The Galactic bulge is old, with no evidence for a younger stellar population, as can be verified in the Colour-Magnitude Diagrams by Zoccali et al. (2003), Clarkson et al. (2008), Clarkson et al. (2011), and Gennaro et al. (2015). Whereas Bensby et al. (2013) claims from his sample of microlensed dwarfs towards the bulge, that $22 \%$ of bulge stars can reach ages as young as $5 \mathrm{Gyr}$, this is not confirmed in the CMDs. A counter-argument on this is given by Haywood et al. (2016). Nataf (2016a) critically reviews age determinations in bulge stellar populations. He concludes that there is a consensus that metal-poor stars are old, whereas for metal-rich stars, there are discrepancies among authors. In particular, a higher helium abundance appears to better fit the red giant branch
(RGB) bump than a younger age. Note that there are young stars in the innermost parts of the bulge, such as the Nuclear Star Cluster (NSC) (Bland-Hawthorn \& Gerhard 2016 and references therein), and the recently revealed Cepheids identified as a young inner thin disk, that are confined in vertical extent (Dékány et al., 2015). These young stars can be formed from mass loss from bulge stars, or else from the dynamical evolution of the bar. However, for most bulge regions, there are no significant numbers of young stars.

Evidence available from kinematics and Metallicity Distribution Function (MDF) are discussed by Babusiaux (2016) and Ness \& Freeman (2016). It is clear that metal-rich stars with $[\mathrm{Fe} / \mathrm{H}]>-0.5$ form the $\mathrm{X}$-shape bulge, corresponding to the bar. There remains as an open question as to whether the more metal-poor stellar population belongs to an old spheroidal bulge population, or to the thick disk and/or halo. The fraction of stars included in different stellar populations is also different among authors: Babusiaux et al. (2010, 2014), Hill et al. (2011), and Gonzalez et al. (2015) consider that there are two stellar populations in roughly equal numbers. Half of them would be the metal-rich X-shaped structure, and the other half belonging to an old spheroid. Ness \& Freeman (2016) and Ness et al. (2013) propose a 5population distribution, with only $5 \%$ of stars to be identified with an old spheroid or inner halo or metal-weak thick disk. From kinematics of Red Clump (RC) stars and M giants, there is evidence for cylindrical rotation, which supports bulge formation from a bar (Kunder et al., 2012; Ness et al., 2013). An 
important caveat is that a few tracers can be biased: RC stars and $\mathrm{M}$ giants are characteristic of metal-rich populations, and would not trace all stellar populations in the bulge. Babusiaux et al. $(2010,2014)$ instead found, from RGB stars, that the metal-poor population is compatible with an spheroid. Di Matteo (2016) suggests that a small spheroidal component, if present, would be maximal in the innermost regions of the bulge. There are also controversial conclusions on the space distribution of RR Lyrae, that are found to have a metallicity peak at $[\mathrm{Fe} / \mathrm{H}] \sim-1.0$. Pietrukowicz et al. (2015) found that they have a triaxial ellipsoid shape, compatible with a bar. Gran et al. (2016) found instead a centrally concentrated spheroidal distribution.

\subsection{Alpha-elements}

McWilliam (2016) reports the available data on chemical abundances in bulge stars. In particular, the abundances of alpha-elements $\mathrm{Mg}, \mathrm{Si}, \mathrm{Ca}, \mathrm{Ti}$, given by Alves-Brito et al. (2010), Gonzalez et al. (2011), Gonzalez et al. (2015), Bensby et al. (2013), Johnson et al. (2014), and Ryde et al. (2016), are enhanced in all fields, showing essentially no difference between different fields. The same applies to Oxygen but there is a larger spread among different authors. The enhancement in alpha-elements indicates that the stellar population was enriched early during bulge formation, due to yields from core-collapse supernovae. A fast chemical enrichment is clearly needed to reproduce these abundances in the Galactic bulge (e.g. Grieco et al. 2015). Ness \& Freeman (2016) and Di Matteo (2016) point out, on the other hand, that the alpha elements in the bulge are similar to those in the (thick) disk, and that all these stellar populations could be the same one.

\subsection{Morphology and simulations}

The Galactic bulge has a boxy/peanut shape (Zoccali et al. 2014; Zoccali \& Valenti 2016). Di Matteo (2016) explains that the formation of the bulge from the bar, accounting only for the thin disk (most common procedure in published work to date), does not reproduce the chemo-kinematic and structural properties of its components. The consideration of the thin plus thick disk in the process is needed, recalling that Snaith et al. (2014) proposed that the thick disk stellar population would involve as much mass as the thin disc.

\subsection{Globular clusters}

Bica et al. (2016) select a sample of globular clusters, and report their properties: metallicity, reddening, space velocity, distance, and abundances. Nataf (2016b) reviews bulge reddening derivation from different indicators, which have implications on distances.

Finally, it is important to note that, according to BlandHawthorn \& Gerhard (2016), the Galactic bulge has a stellar mass of $1.4-1.7 \times 10^{10} \mathrm{M}_{\odot}$, and a total mass of $1.8 \times 10^{10} \mathrm{M}_{\odot}$, in the region covered by the VVV bulge survey (Saito et al. 2012). The Milky Way is accepted to be of SBbc type, but it could be identified to an earlier type, closer to SBb.

\section{REFERENCES}

Alves-Brito, A., Meléndez, J., Asplund, M., Ramírez, I., \& Yong, D. 2010, A\&A, 513, A35

Babusiaux, C., et al. 2010, A\&A, 519, A77

Babusiaux, C., et al. 2014, A\&A, 563, A15

Babusiaux, C. 2016, PASA, 33, 26

Bensby, T., et al. 2013, A\&A, 549, A147

Bica, E., Ortolani, S., \& Barbuy, B. 2016, PASA, 33, 28

Bland-Hawthorn, J., \& Gerhard, O. 2016, preprint (arXiv:1602.07702)

Clarkson, W., et al. 2008, ApJ, 684, 1110

Clarkson, W. I., et al. 2011, ApJ, 735, 37

Dejonghe, H., \& Habing, H. J., eds. 1993, in IAU Symp. Vol. 153, Galactic bulges (Dordrecht: Kluwer)

Di Matteo, P. 2016, PASA, 33, 27

Dékány, I., et al. 2015, ApJ, 812, L29

Gennaro, M., et al. 2015, in ASP Conf. Ser., Vol. 491, Fifty Years of Wide Field Studies in the Southern Hemisphere: Resolved Stellar Populations of the Galactic Bulge and Magellanic Clouds, eds. S. Points, \& A. Kunder (San Francisco: Astron. Soc. Pac.), 182

Gonzalez, O. A., et al. 2011, A\&A, 530, A54

Gonzalez, O. A., et al. 2015, A\&A, 584, A46

Gran, F., et al. 2016, preprint (arXiv:1604.01336)

Grieco, V., Matteucci, F., Ryde, N., Schultheis, M., \& Uttenthaler, S. 2015, MNRAS, 450, 2094

Haywood, M., di Matteo, P., Snaith, O., Calamida, A. 2016, A\&A, in press (arXiv:1606.04092)

Hill, V., et al. 2011, A\&A, 534, A80

Johnson, C. I., Rich, R. M., Kobayashi, C., Kunder, A., \& Koch, A. 2014, AJ, 148, 67

Kunder, A., et al. 2012, AJ, 143, 57

McWilliam, A., 2016, PASA, accepted

Ness, M., et al. 2013, MNRAS, 432, 2092

Ness, M., \& Freeman, K. 2016, PASA, 33, 22

Nataf, D. 2016a, PASA, 33, 23

Nataf, D. 2016b, PASA, 33, 24

Pietrukowicz, P., et al. 2015, ApJ, 811, 113

Ryde, N., Schultheis, M., Grieco, V., Matteucci, F., Rich, R. M., \& Uttenthaler, S. 2016, AJ, 151, 1

Saito, R. K., et al. 2012, A\&A, 544, A147

Snaith, O. N., Haywood, M., Di Matteo, P., Lehnert, M. D., Combes, F., Katz, D., \& Gómez, A. 2014, ApJ, 781, L31

Zoccali, M., et al. 2003, A\&A, 399, 931

Zoccali, M., et al. 2014, A\&A, 562, A66

Zoccali, M., \& Valenti, E. 2016, PASA, 33, 25 